\title{
URUGUAY: ¿TRANSFORMACIÓN O RETORNO?
}

(ENSAYO)

\author{
LAURA DOMÍNGUEZ \\ ELSA GATTI ${ }^{* *}$
}

\begin{abstract}
RESUMEN: En la actualidad política uruguaya existen síntomas contradictorios: centralidad del tema educativo en el discurso oficial y simultáneamente, desarticulación de las líneas de transformación iniciadas en el período 20052010, sustitución de los actores más relevantes que impulsaron la "transformación paradigmática"; mantenimiento de quienes ocuparon lugares destacados en la "reforma educativa" de los 90 y los que impulsaron la actual Ley de Educación, elaborada en círculos cerrados, desconociendo el gran Debate educativo de 2006. Esto, unido al retaceo de la autonomía, y al hecho de que el gobierno está negociando un nuevo préstamo con el BID, nos lleva a plantear que podemos estar ante un "cambio restaurador" de las políticas neoliberales.
\end{abstract}

Palabras clave: Reforma. Transformación paradigmática. Transición.

\section{URUGUAY: TRANSFORMATION OR STEP BACKWARDS?}

\begin{abstract}
Contradictory symptoms mark Uruguay's current political framework. Education has become the central topic of the official discourse while the transformative guidelines implemented between 2005 and 2010 have been taken to pieces. In addition, the most relevant promoters of the "paradigmatic shift" have been kept out whereas the most highlighted players of the 90s "education reform" and those who designed, in inner circles, the present Law of Education, which ignores the huge 2006 Debate on Education are still in command. On top of it, autonomy has been restricted and the government is negotiating a new loan with the Interamerican Development Bank. As a result, we are prone to think that we might be facing a "restoring change" of neoliberal policies.
\end{abstract}

Key words: Reform. Paradigmatic transformation. Transition.

\footnotetext{
* Magíster en Psicología y Educación y docente de Pedagogía a nivel de grado y posgrado en la Universidad de la República (UR), Uruguay. E-mail: dominguez.dom@gmail.com

* Especialista en Pedagogía Universitaria y en Ciencia Política y realidad latinoamericana y docente de posgrado en el Instituto de Perfeccionamiento y Estudios Superiores (IPEs) y en la Universidad de la República (UR), Uruguay. E-mail: elsa.gatti@gmail.com
} 


\section{URUGUAY: TRANSFORMATION OU RETOUR EN ARRIÈRE?}

RÉSUMÉ: L'actualité politique uruguayenne présente des symptômes contradictoires: la centralité du thème éducatif dans le discours officiel et, en même temps, la désarticulation des lignes de transformation mises en œuvre entre 2005 et 2010; et la substitution des acteurs les plus pertinents ayant impulsé la "transformation paradigmatique" alors que tous ceux qui occupaient places de choix dans la "réforme éducative" des années 1990 et ceux qui ont conçu, à huis clos, la Loi sur l'éducation actuelle sans tenir compte du grand débat éducatif de 2006 ont été maintenus à leur poste. Si l'on y ajoute le fractionnement de l'autonomie et le fait que le gouvernement est en train de négocier un nouvel emprunt avec la Banque Interaméricaine de Développement, nous ne pouvons qu'affirmer que nous sommes face à un "changement restaurateur" des politiques néolibérales.

Mots-clés: Réforme. Transformation paradigmatique. Transition.

\section{La apuesta al cambio... entre la ilusión y el desencanto}

$\mathrm{U}$ ruguay registró un cambio sustancial en la orientación de sus políticas educativas a partir del año 2005, cuando, por primera vez el "Frente Amplio - Encuentro Progresista" accedió al gobierno, desplazando a los partidos tradicionales (Partido Nacional y Partido Colorado) que habían gobernado el país desde su constitución como Nación independiente. Estamos viviendo el segundo período de gobierno frenteamplista o "progresista"1 con matices y énfasis diferenciales, que a algunos satisfacen plenamente y a otros decepcionan, por haber creído que el gobierno de José (Pepe) Mujica iba a profundizar y radicalizar los cambios en una línea de izquierda revolucionaria, lo que no se evidencia todavía en el campo de la educación.

La nueva administración educativa, lejos de continuar y profundizar lo que la anterior llamó “una transformación en marcha” (Yarzábal et al., 2010) está haciendo borrón y cuenta nueva, al tiempo que está retomando líneas que habían sido desarrolladas en la década de los 90 y ampliamente cuestionadas por la izquierda. La demora en designar las nuevas autoridades, así como el desconocimiento que éstas han hecho de las "líneas transversales de transformación" impulsadas en el período 2005-2010, desmantelando los equipos técnicos que las diseñaron y llevaron adelante, es una prueba de ello. Ejemplos claros de esto son la desactivación de las Comisiones de Educación Sexual y de Propuesta Educativa. Esta última había realizado, luego de una consulta a nivel nacional, la fundamentación exhaustiva de un Plan Nacional de Educación 2010-2030, que debería ser elaborado luego de un amplio debate. Esta última etapa ha quedado en suspenso.

El discurso oficial sigue afirmando la centralidad que al tema Educación le dio desde su asunción el Presidente de la República. Una muestra de dicha prioridad 
parecería ser el hecho de que el primer Consejo de Ministros ampliado (febrero 2011) fue precisamente con las autoridades de la educación. Sin embargo, este hecho es señal, no tanto de la centralidad del tema, sino más bien de la tramitación de la cuestión educativa desde el Poder Ejecutivo, hecho que es preocupante porque afecta la autonomía constitucional de los Entes de la educación.

Actualmente éste es un asunto "urticante" en los medios de comunicación, que se hacen eco y aprovechan la ambigüedad de los discursos políticos, centrados en la necesidad de aumentar la cobertura (en especial en Educación Media donde los índices de abandono son preocupantes) ${ }^{2}$ mejorar la "calidad" de la educación que se imparte, y aumentar el "control" de la misma desde los órganos políticos (Poderes Ejecutivo y Legislativo), llegando a "los límites de la autonomía" establecidos en la Constitución de la República. ${ }^{3}$

La problemática recurrente en los discursos de política educativa en la actualidad, remite a la escasa retención del sistema educativo, especialmente a nivel de Educación Media.

La "cuestión docente" aparece como una variable explicativa de este "fracaso" del sistema. Se continúa con la perspectiva estadística del análisis y es así que se acumulan datos sobre repetición y abandono ${ }^{4}$ así como resultados de evaluaciones internacionales de los aprendizajes, (especialmente los obtenidos en las pruebas de lo Programme for International Student Assessment (PISA). ${ }^{5}$ Se recorre de esta manera un camino ya iniciado en periodos anteriores al primer gobierno progresista, que llevaba a la "demonización" de los profesionales de la educación. Se vuelve a una visión propia de periodos que otrora se criticaban.

Juan Carlos Tedesco sostenía en el prólogo del estudio “Los docentes uruguayos y los desafíos de la profesionalización":

La década de los años ' 90 ha sido un periodo de profundas transformaciones educativas en la mayor parte de los países de América Latina. El balance de los resultados de este proceso muestra avances importantes en términos de aumento de cobertura, modificaciones en los estilos de gestión y administración, renovación de los contenidos curriculares e incorporación de nuevas tecnologías. Los resultados son menos satisfactorios, en cambio, cuando se observan los logros de aprendizaje de los alumnos. Si bien es cierto que los logros de aprendizaje son la consecuencia de factores muy diversos y complejos, es plausible sostener que una de las explicaciones del bajo impacto de las reformas en los procesos de enseñanza-aprendizaje radica en el "factor docente", entendido como conjunto de variables que definen su desempeño (...). (Tedesco, 2003, p. 9)

En la presentación de ese mismo estudio el entonces Director Nacional de Educación, Javier Bonilla sostenía "la inexistencia de señales claras de la presencia de lo que se ha dado en llamar "malestar docente" (Bonilla, 2003, p. 12), lo que ha sido desmentido en una investigación realizada por los sindicatos de la enseñanza 
con el apoyo del Consejo Latinoamericano de Ciencias Sociales (Clacso) (CTERA et al., 2005).

\section{Más allá de los énfasis, los paradigmas en juego}

Dada la situación planteada en el apartado anterior, cabe preguntarse: la política educativa puesta en marcha en el actual gobierno, ¿se aparta o se confunde con la política neoliberal que en los discursos intenta superar?

Resulta oportuno entonces, hacer un breve recorrido histórico de lo que ha ocurrido en materia educativa en las últimas décadas.

Esther Mancebo (2007) presenta la siguiente periodización de las políticas educativas en el Uruguay a partir de la restauración de la democracia:

- Restauración (1985-1990)

- Acumulación diagnóstica (1990-1995) ${ }^{7}$

- Reforma (1995-2000)

- Amortiguación incremental (2000-2005)9

- $\quad$ Debate educativo (2005-2006) ${ }^{10}$

La autora señala la "encrucijada" ante la que se encontraba el sistema educativo en el momento que realiza su periodización: entre "la inercia, el cambio restaurador o el cambio innovador" (Mancebo, 2007, p. 36).

Lo que parece interesante analizar aquí es qué implican las caracterizaciones del párrafo anterior, o sea qué es lo que está implícito cuando se habla de "cambio restaurador" y "cambio innovador". Restaurador y/o innovador con respecto a qué. En medio de la periodización que realiza Mancebo, se encuentra la "Reforma" que tuvo la impronta de las políticas neoliberales de los años noventa. ¿De qué hablamos entonces cuando utilizamos los términos restauración y/o innovación para referirnos a las políticas actuales?

Recordemos cuáles eran las prioridades de las líneas de política educativa de aquella década. J.C. Navarro (2006), en un trabajo en la línea del BID distingue dos líneas de política educativa efectivas:

1. De expansión y aumento de la matrícula.

2. De mejoramiento de la calidad y la eficiencia.

Según este autor, la política de incentivos y evaluación docente, que correspondería a la política de mejoramiento de la calidad y eficiencia, es de tipo 
básico "porque toca la esencia misma de la economía de la educación" (Navarro, 2006, p. 6)

Asimismo, señala que las políticas de expansión son las que generan mayor acuerdo, en tanto las otras son motivo de tensiones, de conflictos y resistencias sobre todo por parte de los docentes. Desde su perspectiva, el escenario tiende a estar protagonizado por las corporaciones docentes, y otros actores sociales no suelen hacer oír su voz.

Este discurso recuerda el que impregnó la reforma educativa de los 90 ("reforma Rama") que, si bien tuvo en Uruguay matices diferenciales respecto a otros países del continente (como por ejemplo la fuerte apuesta a la educación pública), incorporó en la conducción de la misma, criterios empresariales, tal como lo exigían los organismos internacionales de crédito, en cuyos préstamos y asesorías se apoyó.

En ese sentido la "reforma educativa" fue continuadora de las políticas neoliberales instauradas en el país durante la dictadura cívico-militar (1973-85).

Es necesario recordar que el periodo de reformas que se inició en la década de los noventa, se orientó a deslegitimar sistemáticamente a los docentes como profesionales capaces de incidir en las políticas educativas. Esto se hizo incorporando otros actores (sociólogos, economistas, entre otros) a los que se calificaba de "expertos", que convivieron paralelamente con un sistema de privilegios en las remuneraciones y con alta incidencia en la marcha de las políticas educativas.

Al iniciarse el primer gobierno progresista, la línea de análisis desde la Administración Nacional de la Educación Pública (ANEP) ${ }^{11}$ fue otra. Acerca de la misma dieron cuenta los equipos técnico-docentes asesores del Director Nacional de Educación dr. Luis Yarzábal. ${ }^{12}$ Uno de ellos ${ }^{13}$ elaboró en 2005 un documento en el que se sostiene que:

- El período postdictatorial - cuatro mandatos presidenciales, los dos últimos asociados a la llamada "reforma educativa" - fue de gran frustración:

- Los procesos negativos iniciados durante la dictadura no fueron revisados ni cuestionados suficientemente y, en algunos casos, se profundizaron.

- La reforma estuvo revestida de autoritarismo. Rehuyó todo debate social y profesional sobre el futuro de nuestra educación nacional. Desconoció y desnaturalizó las prácticas democráticas, participativas y autonómicas propias de nuestro acervo cultural.

- La segmentación social de los centros educativos contribuyó al afianzamiento creciente de la desigualdad, con considerables ventajas para el sistema privado.

- Se acentuó la decadencia de la calidad y el prestigio de la educación pública; proceso ligado, entre otras causas, a la pérdida de protagonismo de las discusiones específicamente pedagógicas. 
- Se agravó el deterioro de las condiciones de trabajo y de los salarios docentes.

- Se perdió la dimensión temporal del ser humano al privarse a los educandos de las categorías propias del modo de pensar histórico. Se afecta así su derecho a situarse en el tiempo.

- Se renunció al perfil cultural autónomo que había configurado la identidad y calidad de nuestra educación nacional, encarnado en nuestros docentes, y se adoptaron criterios gerenciales tecnocráticos. La evaluación de la educación privilegió a los objetivos mensurables en desmedro de la discusión de otros fines educativos.

- Se profundizó la burocratización del sistema educativo y se creó una superestructura técnica parcialmente superpuesta a la organización del sistema educativo nacional.

- Se recurrió a fuentes de financiamiento externo, incrementando la deuda externa, en desmedro de la soberanía nacional. (Abero et al., 2007)

El vuelco que se operó en la administración de la educación en el primer período de gobierno del Frente Amplio (2005-2010), desde nuestro punto de vista tuvo dos ejes de alta gravitación política que apuntaron a revertir los fenómenos descriptos por el equipo asesor:

1. La participación democrática, tanto a través de la actuación de docentes en los consejos y comisiones con delegados de las Asambleas TécnicoDocentes (ATD), ${ }^{14}$ como la participación de los estudiantes, docentes y la sociedad civil en su conjunto en lo que fue el Debate Educativo y el Congreso Nacional de Educación “Maestro Julio Castro” (2006).

2. El proceso de institucionalización de programas de mejoramiento, que hasta entonces funcionaban paralelos al sistema y respondían a políticas vinculadas a las directivas de los organismos multilaterales de crédito.

El dr. Luis Yarzábal (2010) entendía que se estaba en un periodo de transición paradigmática:

La nueva administración llegaba dispuesta a superar el paradigma educativo vigente en aquel momento, para pasar a otro propio de una sociedad que había optado por impulsar una estrategia de desarrollo sostenible basada en el crecimiento económico orientado a generar igualdad de oportunidades y asegurar justicia social. Pero queríamos conducir esa transición sin ocasionar incertidumbres ni traumatismos que siempre producen daños irreparables en el proceso de formación de quienes deben sufrirlos. Especialmente si tenemos en cuenta que la educación es una de las funciones de la sociedad cuyos cambios producen efectos (tangibles y, sobre todo, intangibles) cuyas consecuencias no son visualizadas sino en el mediano y largo plazo, cuando ya puede ser tarde para corregir eventuales errores estratégicos. (Yarzábal, 2010, p. 18)

La política educativa se orientó entonces hacia lo que la entonces consejera Lilián D’Elía (2010) denomina "liderazgo democrático-transformador": "Ya en 2005 planteábamos la necesidad imprescindible de la participación y el manejo 
democrático de la autoridad como elementos fundantes de todas las otras líneas estratégicas de la ANEP" (D’Elía, 2010, p. 51).

D'Elía vincula directamente los conceptos de participación democrática, bien público y autonomía de las instituciones educativas.

Recuerda que nuestra Constitución, al reconocer y consagrar la autonomía de la educación pública, recoge una rica tradición vinculada a las luchas por la laicidad, que llevaron a autonomizar la educación de toda dependencia respecto a los fines e intereses privados de cualquier credo religioso o corriente ideológica, incluida la de los partidos políticos que pudieran acceder al gobierno, poniendo así el interés público, representado por el Estado, por encima de las razones privadas de los funcionarios y de los organismos de gobierno.

Esta tradición, tiene su origen y quizás su expresión más brillante en las obras de José Pedro Varela (1874-1876). Como contracara de esta política surgida en el marco del Ente autónomo de la educación ANEP - entre cuyas autoridades se encontraban tanto Yarzábal como D’Elía -, en el seno del propio gobierno progresista, se erigía simultáneamente otra tendencia que emanaba del Ministerio de Educación y Cultura (MEC). Puede decirse entonces que en el periodo 2005-2010 ya comenzaron a chocar dos líneas de política educativa en el marco del gobierno progresista, aunque podría decirse que logró imponerse la de la ANEP. El maestro Miguel Soler Roca (2010) entiende que la tradición autonómica se avasalló sin embargo en el trámite dado a la actual Ley de Educación n. 18.437, tanto en su etapa de formulación en la órbita del MEC como en su casi nula discusión en el Parlamento, en el 2ª semestre de 2008.

\footnotetext{
Una valoración general del trabajo realizado en este período me lleva a usar un categórico adjetivo: decepcionante. El país acababa de experimentar la conmoción positiva de un debate nacional abierto y pasó a una fase de trabajo concentrada fundamentalmente en el equipo del MEc. La idea de abordar la tarea de manera participativa, representativa y consultiva fue dejada de lado. Sé que los voceros del Ministerio arguyen lo contrario, detallando las instancias en que sometieron sus propuestas a organismos de la administración educacional pública, a sindicatos docentes o a especialistas en derecho constitucional. Lo siento de verdad; el seguimiento detallado de esta etapa me lleva a la conclusión de que los contactos realizados - asistemáticos, no resultantes de un compromiso colectivamente asumido y carentes de una voluntad compartida de situar el interés de los destinatarios de la función educativa por encima de las divergencias por el poder institucional - tuvieron lugar más por la presión de entidades externas al Ministerio que por iniciativa de éste. Un ejemplo revelador: los sindicatos de docentes se enteraron por la prensa de la existencia de un anteproyecto de ley y tuvieron que pedir acceso a él para poder opinar, a posteriori de su elaboración. (Soler Roca, 2010, p. 302-303)
}

Si a esto se agrega la tramitación en el Parlamento a marcha camión; la no convocatoria de la ANEP a la Comisión de Educación del Senado (que es preceptiva constitucionalmente) y su aprobación en tiempo récord por la simple razón de que se cerraba el período legislativo, el resultado ha sido un profundo malestar que vino 
a confirmar la sospecha de los sindicatos docentes y estudiantiles de que la Ley ya estaba "cocinada" y que uno de sus ingredientes básicos era la ampliación de la influencia del MEC, que ahora pasa a controlar ámbitos educacionales que no le son atribuidos por la Constitución (educación inicial, no-formal, de adultos) en un claro avasallamiento de las competencias y autonomías de los Entes de la enseñanza. Este malestar fue muy bien aprovechado por la oposición para deslegitimar tanto el trámite como el contenido de la ley, y de paso, todo el accionar de la ANEP, sus políticas y sus logros (Gatti, 2010).

Pero quizás lo más grave, es que la frustración de las expectativas generó una desmovilización, que se traduce, entre otras cosas, en una renuncia de hecho a todo debate de fondo sobre los temas educativos.

Nos parece importante entonces reafirmar que la transición paradigmática de la que hablaba Yarzábal (y que se percibe por lo menos estancada) no es ni más ni menos que el tránsito desde un sistema autoritario, regido por el mercado, hacia uno democrático, que concibe a la educación como derecho de todos, constructora de personalidades autónomas y ciudadanos libres, críticos y solidarios. Esto hace a la filosofía de la política educativa, así como a la construcción de programas que apunten a la consolidación de mecanismos de participación legítimos y no meramente legitimantes del status quo.

\section{Continuidades, quiebres y retornos}

A pesar de la continuidad del gobierno frenteamplista o "progresista", es de destacar que la transición entre el primero y el segundo mandato supuso costos a las políticas iniciadas y quizás "traumas institucionales".

Salvo en lo que respecta al Plan Ceibal "un niño, una laptop" del periodo Vázquez, y a la extensión de la cobertura preescolar así como del tiempo pedagógico (fundamentalmente a través del modelo de "Escuelas de Tiempo completo", esgrimidas como buque insignia del discurso electoral del Presidente Mujica), provenientes de la Reforma de los noventa (esas políticas de expansión sobre las que hay mayor consenso, al decir de Navarro), la sensación es que nada de lo hecho durante el periodo 2005/2010 es retomado fuertemente en el segundo gobierno frenteamplista. ${ }^{15}$

En la educación se vivió un cambio con la nueva administración que no permite visualizar procesos de transición. Quizás sí podríamos aventurar que nos encontramos ante lo que se ha denominado "cambio restaurador" pero esta vez de lo que fueron las políticas de los noventa. Lo que es señal, por lo menos de una tensión en la interna de la fuerza progresista, donde, en cuestiones de educación, los 
cambios contradicen el progresismo que se suele sostener en palabras. El encargo es poner en práctica los lineamientos programáticos de una ley (la n. 18.437) que no satisface a nadie, no resuelve los problemas sustantivos del sistema, desconoce la voluntad popular expresada en el Debate del 2006 y se centra en delinear aspectos puntuales que no tendrían que integrar el texto de una ley, sino quedar librados a las decisiones autónomas de las autoridades educativas.

A pesar de los informes de gestión de la administración anterior, de la fundamentación conceptual y filosófica de las líneas emprendidas; a pesar incluso del laborioso y discutido proceso de elaboración de un documento que permitiera empezar a considerar un plan prospectivo al 2030 (en el marco de la ENIA ${ }^{16}$ ), los énfasis son otros. No hubo transición.

Cada vez es más fuerte el papel del Ministerio de Educación y Cultura, lo que da una señal de la perspectiva, avasalladora de la autonomía, que se ha afianzado.

Aquí nos encontramos ante la tensión entre legalidad y legitimidad.

De acuerdo a la Ley de Educación n. 18.437 (2008), los Consejos Directivos de la enseñanza no universitaria cuentan con miembros designados por el Poder Ejecutivo previa venia del Senado, y otros electos directamente por los docentes. A estos últimos, los legisladores de la oposición les llaman "delegados gremiales", lo que les permite deslegitimarlos en nombre del "corporativismo" de que hablaba Navarro - y del que se hace eco la prensa - reclamando esos lugares para representantes políticos de la oposición, argumentando que ellos son los auténticos representantes del "soberano". Reclamo que el gobierno "progresista" no discute. Pasa entonces a la anécdota la experiencia popular del Congreso "Maestro Julio Castro" y adquiere una función monopólica la comisión interpartidaria de educación que elabora un documento donde establece las prioridades educativas. Aparece como interlocutor de la ANEP el Consejo de Ministros perdiendo espacio el ámbito parlamentario.

En el "Consejo de Ministros ampliado" al que asistieron los integrantes de la Comisión Coordinadora del sNep, la atención estuvo centrada en los problemas de la Educación Media, para los que, el Presidente del Codicen, dr. José Seoane presentó cinco propuestas de innovación:

- "Construcción de espacios abiertos de cooperación educativa": impulsar que los liceos se relacionen con otras instituciones no necesariamente educativas.

- "Articulación socialmente valiosa entre el mundo del estudio y el mundo del trabajo".

- "Creación de Instituciones de Educación Media", que reúnan la educación técnica y la general. 
- "Superación en logros y equidad de los aprendizajes", para lo cual se implementarán los programas Pro-Lee y Pro-Razona (Programas de fortalecimiento del dominio de la lengua y del razonamiento lógico-matemático, respectivamente).

- "Instituciones amigables y protagónicas", para mejorar el vínculo con los alumnos y la comunidad. ${ }^{17}$

Algo que debe ser considerado es cómo se vehiculizan estas supuestas innovaciones. $^{18}$

Es curioso que, en la reunión, haya jugado un papel protagónico el único Consejero de la oposición, que está sacando partido de la desorientación oficial. Un ejemplo paradigmático de esa desorientación es la relevancia de la proyectada política de incentivos a estudiantes. El mero cambio en los vocablos utilizados: dejar de hablar de "becas" para pasar a hablar de "incentivos" supone un cambio importante, que es un retorno al paradigma economicista de los 90 .

Aunque podrían considerarse sinónimos en la medida en que una beca se constituye en un incentivo, las lógicas que sostienen una y otra categoría son diferentes. El incentivo, como estímulo o como excitación para hacer algo, en este caso "estudiar" se constituye en la contracara de la idea de educación como derecho. El eje del deseo deja de estar en el aprender y pasa a estar centrado en la dotación económica para seguir los estudios.

Otro discurso preocupante es el que pretende contraponer los derechos de los estudiantes a los de los docentes, convirtiendo a estos últimos en los chivos expiatorios de la "crisis educativa". "Toda la legislación escolar está basada más en los derechos de los docentes que en los derechos de los educandos (...) entonces, ¿Seguimos pensando en la necesidad de los centros educativos o en los derechos de los docentes?" (Entrevista a Luis Garibaldi, en: López Belloso, 11 feb. 2011, p. 4).

Con este concepto alude Garibaldi, ${ }^{19}$ a uno de los problemas claves en Enseñanza Secundaria: la forma de elección de horas docentes, que entiende que debe pasar a ser, al igual que en Educación Primaria, elección de cargos, para radicar a los docentes en los centros educativos y evitar el "docente taxi". Totalmente compartible, si no fuera porque resulta insostenible pensar que la actual modalidad de elección, que genera incertidumbre e inestabilidad laboral año a año, sea algo que esté funcionando en beneficio de los derechos de los docentes.

Polarizar con este ejemplo derechos de estudiantes versus derechos de docentes, parece por lo menos simplificador cuando no mal intencionado.

Que en la misma edición de Brecha, en otra entrevista, el Vicepresidente de la República insista en que el sistema está pensado más a favor de los docentes que 
de los estudiantes, es sintomático de un leit motiv del gobierno. Y lleva directamente a la cuestión de los cambios curriculares y la evaluación docente. "Para el país es fundamental que el funcionamiento del sistema educativo público enfatice en el alumno y no en el docente. Para el gobierno nacional es fundamental que haya una modernización de los contenidos, ese es un lineamiento" (Entrevista a Danilo Astori, en: López Belloso, 11 feb. 2011, p. 7).

En el discurso oficial la "modernización de los contenidos" está centrada en la idea de que hay que enseñar cosas "que sirvan" a los muchachos, sobre todo a la hora de buscar empleo.

Así, por ejemplo el Senador oficialista Cr. Alberto Couriel sostiene que:

La inserción económica internacional requiere de la competitividad, que tiene carácter sistémico, para lo que es central el progreso técnico, la calificación de los recursos humanos, tanto empresariales como de trabajadores calificados y por lo tanto depende directamente del sistema educativo. Pero este debe adaptarse a los requerimientos del mercado, de la estrategia productiva, de las características de la estructura productiva. Porque los recursos humanos calificados tienen que conseguir empleos productivos adecuados.

Lejos de avanzarse entonces hacia la "integralidad de la educación", lo que se está produciendo es una inversión de las prioridades, en una dirección que es la exigida por el mercado. Por algo el Ministro de Educación y Cultura ha iniciado una serie de contactos e intercambios con su par de Chile, laboratorio del neo-liberalismo. ${ }^{20}$

Por eso planteábamos en el título de este artículo, la dolorosa interrogante de si estamos asistiendo a una "transformación" o a un "retorno" a las directrices que se impusieron desde afuera y desde arriba en los 90, causando estragos en el sistema educativo nacional.

\section{(In)conclusiones}

En el 2010, Nicolás Bentancur (2010) auguraba “(...) es previsible un 'policy making' complejo, cruzado por las múltiples contradicciones y antagonismos expuestos. La pregunta fundamental para descifrar su impronta - que todavía no puede ser respondida - refiere a qué ámbito se constituirá en el verdadero centro de gravedad de las políticas educativas" (Bentancur, 2010, p. 21).

Si las cuestiones relativas a las políticas de calidad y eficiencia son hoy - en los círculos políticos y en la opinión pública - las relevantes y más urticantes, es en parte, por el contenido que se le da a estos conceptos, extrapolados del campo económico, que asimilan la educación con un bien o servicio, y no con un derecho humano fundamental. 
La eficiencia en los procedimientos, el éxito en el cumplimiento de "metas" traducidas en estándares descontextualizados y homogeneizantes, "evaluables" a través de la medición externa de indicadores en los que los "resultados" habilitan la clasificación y el ordenamiento de personas, de instituciones y de países, están comenzando a impregnar nuevamente el discurso y las prácticas educativas, con grave lesión para la soberanía nacional, ya que, quien decide la forma de evaluar está sobredeterminando lo que se debe enseñar, los currículos, contenidos y métodos de enseñanza.

Por eso preocupa que el Presidente de la República haya firmado en Mar del Plata las "Metas 2021. La educación que queremos para la generación de los Bicentenarios" - impulsadas por CEPAL, SEGIB y OEI - sin previa consulta y aprobación de las autoridades autónomas de la educación nacional.

A esto se agrega que el gobierno está negociando un nuevo préstamo con el BID, para el mejoramiento de la Educación Media y la Formación Docente. El nuevo Programa, PAEMFE ${ }^{21}$ - cambio de sigla, pero no de orientación respecto a los que se suscribieron en los 90 -, habría sido elaborado por un equipo de "expertos líderes", que plantean en el documento a ser firmado que "dos son los desafíos actuales: aumentar el acceso y la retención al sistema y mejorar la calidad educativa, tanto en los niveles de aprendizaje como en el conocimiento y las competencias para desempeñarse en las áreas que la sociedad actual requiere". ${ }^{22}$

Se advertirá la recurrencia en el enfoque que hace un lustro planteaba Navarro.

El discurso a nivel oficial pone el énfasis en la necesidad de reforzar la educación técnica y la formación de "recursos humanos calificados" para el desarrollo del país productivo. Sin embargo, el programa de gobierno del Frente Amplio era -valga la redundancia - más amplio: apuntaba al desarrollo del "país productivo con justicia social". Y para ello, la educación debía ser democrática, participativa e integral.

Si bien los distintos actores sociales reconocen la complejidad de la situación, se insiste en recurrir a los incentivos como camino para la superación de la crisis: incentivo a docentes por desempeño (ya implantado durante la reforma educativa de los 90), al que se quiere agregar ahora el incentivo económico a estudiantes para que permanezcan o se reinserten en el sistema.

Sostenemos entonces, que a pesar del proceso iniciado en 2005 que tuvo como sello de impacto inicial el Congreso Nacional de Educación "Maestro Julio Castro", en las políticas educativas de los dos gobiernos progresistas, se mantuvo una tensión entre dos líneas que al momento actual se dirime a favor de una que no se aparta, sino más bien se confunde con la política neoliberal que en los discursos se intenta 
superar, que deslegitima y desplaza a los profesionales de la educación, mientras va transfiriendo poder de decisión educativa a los partidos políticos.

\section{Notas}

1. Primer período: 2005-2010; segundo período: 2010-2015.

2. Las últimas encuestas e investigaciones revelan que menos de un $70 \%$ culmina el Ciclo Básico obligatorio, y no llega a un $40 \%$ el porcentaje que culmina la Educación Media Superior. Un 16,7\% de los jóvenes de entre 14 y 24 años no estudia ni trabaja (ENAJ, 2008; ECH, 2009; UNICEF, 2011).

3. Palabras del Ministro Dr. Ricardo Ehrlich. Los límites hacen referencia a que, por disposición constitucional, la Educación Pública en Uruguay está dirigida por "uno o más Consejos Directivos Autónomos" (art. 202 de la Constitución de la República), que no tienen relación de dependencia respecto al Ministerio de Educación y Cultura (MEC).

4. Anuario estadístico del MEc (2009).

5. Por haber cuestionado la validez de estas evaluaciones internacionales y haber hecho propuestas que se apartaban del discurso "políticamente correcto", la oposición ha pedido la renuncia o la destitución de un Consejero de Enseñanza Secundaria, electo por sus pares.

6. Quizás el punto más destacado de este proceso de restauración haya sido la creación de la Comisión de Concertación Programática, con amplia representación política y sindical, que elaboró la Ley n. 15.739, conocida como "Ley de emergencia", aunque se mantuvo vigente por más de 20 años.

7. Es el período de los estudios de la CEPAL, que catapultaron a Germán Rama, quien luego fue designado Director Nacional de Educación y desde allí impuso la "Reforma Educativa" que domina el siguiente período.

8. Mancebo, en su periodización, al referirse al periodo de Reforma señala que "Desde el punto de vista procesual se trató de una reforma racionalista (...) que buscó fortalecer las instituciones existentes de forma tal que permitieran a los actores un funcionamiento adecuado en el marco de un modelo 'clásico' de política educativa, según el cual las policies derivan su legitimidad del carácter democrático de las autoridades educativas, quienes son responsables de la elaboración de las políticas educativas y de la organización del servicio, al tiempo que los profesores se ubican como 'productores' del servicio y los estudiantes como 'receptores' del mismo" (Mancebo, 2007, p. 11).

9. No se registran cambios significativos en las políticas educativas respecto al período anterior. Los procesos iniciados durante la "Reforma" continúan, pero perdiendo agresividad.

10. Quizás la expresión "Debate Educativo" no sea suficientemente consistente, pero alude al momento fundacional que representaron el "Debate Educativo Nacional" y el "Congreso Nacional de Educación "Maestro Julio Castro" en el 2006, ambos con amplia participación ciudadana.

11. La ANEP es un Ente Autónomo que tiene bajo su órbita toda la educación pública no universitaria (Pre-escolar. Primaria, Secundaria, Técnico-profesional y Formación Docente), aunque cada nivel tiene un Consejo Directivo con relativa autonomía respecto al órgano central.

12. Presidente del Consejo Directivo Central (CODICEN) de la ANEP en el período 2005-2010

13. Este equipo estuvo integrado por los Prof. Beatriz Abero, Rosario Beisso, Mauricio Langón y el Maestro Miguel Soler Roca (Dr. HC de la UR).

14. Las ATD, establecidas por Ley n. 15.739 en 1985, están integradas por todos los docentes del Sistema Nacional de Educación Pública (SNEP); tienen carácter consultivo y participan con voz en los Consejos directivos de cada nivel de la educación. 
15. Por otra parte, el plan Ceibal no fue producto de una elaboración participativa y autónoma como sí lo fueron otras experiencias como las reformas curriculares en todos los niveles de la educación pública, o los Posgrados docentes en el Área de Perfeccionamiento y Estudios Superiores (APES), con comités académicos en los que participaban delegados de las ATD, de los Consejos Desconcentrados y de la Universidad de la República (UR).

16. Estrategia Nacional para la Infancia y la Adolescencia.

17. El documento presentado puede consultarse en: <www.anep.edu.uy $>$.

18. En diciembre 2010 el Consejo Directivo Central de la ANEP aprobó un "Proyecto de Fortalecimiento de las Instituciones para la mejora educativa" (PROFIME) y creó una comisión para llevarlo adelante, coordinada por el único Consejero que representa a la oposición, encargándole hacer un Plan orgánico de implementación, que incluya nombres para integrar un Consejo Consultivo (Res. n. 64, Exp. 1-519/11; Acta 95). Los nombres que han sido propuestos son, en su casi totalidad, ex jerarcas y colaboradores de la Reforma Rama. La propuesta en discusión se basa en la idea que "el centro educativo es el eje de la innovación y la mejora (efecto establecimiento, movimiento escuelas eficaces, etc.)" (¿modelo Chile?) y el "entrenamiento" de los equipos docentes por un sistema de "coaching".

19. Director de Educación del MEC en los dos períodos frenteamplistas; uno de los pocos "sobrevivientes", que juega un papel muy protagónico en la reorientación de las políticas y la elaboración de los marcos legales de las mismas.

20. Ante las críticas recibidas, el Ministro Ehrlich ha cambiado su discurso, diciendo que son dos modelos muy distintos pero que han obtenido similares resultados en Pisa, por lo que es interesante compararlos y eventualmente aprender cosas uno del otro.

21. Programa de Apoyo a la Educación Media y a la Formación en Educación.

22. Diario La Juventud, 23 feb. 2011, que remite al sitio web del BID: <www.bid.org.uy>.

\section{Referencias}

ABERO, B. et al. Algunos conceptos y propuestas sobre educación. Montevidéo: Grupo de Consulta Educativa, 2007. Disponible en: <www.anep.edu.uy>

ADMINISTRACIÓN NACIONAL DE EDUCACIÓN PÚBLICA. Documento de la ANEP para el primer encuentro con el Presidente de la República y el Consejo de Ministros. Montevideo: ANEP, 14 feb. 2011.

ANEP-CODICEN. Una transformación en marcha: políticas instrumentadas por el CoDICEN 2005-2009. Montevidéo: ANEP, 2010.

BARRERO ALVAREZ, M.D. Al MEMFod de Rama lo suplanta el PAEMFE progresista. La Juventud, Montevideo, 23 feb. 2011.

BENTANCUR, N. Las políticas educativas tras el primer gobierno de izquierda en Uruguay: ¿Transformación o reforma?; ponencia presentada al Tercer Congreso Uruguayo de Ciencias Políticas. Montevideo: AUCIP, 2010.

BONILLA, J. Conocer a los docentes: un cambio hacia la profesionalización. In: ANEP/ unesco/IIPE. Los docentes uruguayos y los desafíos de la profesionalización. Montevideo, 2003. 
CTERA et al. Las reformas educativas en los países del Cono Sur: un balance crítico. Buenos Aires: ClACSO, 2005.

COURIEL, A. Crecimiento y distribución. La República, Montevideo, 23 mar. 2011

D’ELÍA, L. Líneas fundamentales de la conducción democrática y participativa de la ANEP. In: ANEP-CODICEN. Una transformación en marcha: políticas instrumentadas por el CODicen 2005-2009. Montevideo: ANEP, 2010.

DOCUMENTO DE ACUERDOS SOBRE EDUCACIÓN: Frente Amplio, Partido Nacional, Partido Colorado, Partido Independiente. Montevideo, 11 mayo 2010.

EL IMPULSO contra el freno; entrevista con el Director de Educación Luis Garibaldi. Brecha, Montevideo, p. 4, 11 feb. 2011.

GATTI, E. Presentación del libro Una transformación en marcha. Revista Voces, Montevideo, n. 33, p. 47-56, abr. 2010.

LEYES DE EDUCACIÓN n. 14.101 (1985) y 18.437 (2008).

LÓPEZ BELLOSO, R. Entrevista con el Vicepresidente Danilo Astori. “Es muy peligroso el oportunismo tributario". Brecha, Montevideo, p. 6-7, Política, 11 feb. 2011.

MANCEBO, M. La educación uruguaya en una encrucijada: entre la inercia, la restauración y la innovación. In: BentANCuR, N. (Coord.). Las políticas educativas en Uruguay: perspectivas académicas y compromisos políticos. Montevideo: MEC, 2007.

NAVARRO, J.C. Dos clases de políticas educativas. In: IPEs/BID. La política de las políticas públicas: progreso económico y social en América Latina; informe 2006. [s.L.]: BID, 2006. (Cap. 10). (Serie Preal documentos, n. 36).

SEOANE, J. Cinco orientaciones básicas como propuestas para la transformación de la EM. 2011. Disponible en: <www.anep.edu.uy>

SOLER ROCA, M. El debate educativo y la Ley 18.473. In: ANEP-CODICEN. Una transformación en marcha: políticas instrumentadas por el CODICEN 2005-2009. Montevideo: ANEP, 2010.

TEDESCO, J.C. Prólogo. In: ANeP; unesco; IIPE. Los docentes uruguayos y los desafíos de la profesionalización. Montevideo: ANEP, 2003.

URUGUAY. Ministerio de Educación y Cultura. Anuario estadístico 2009. Montevideo: IMPo, 2010.

URUGUAY. Plan Nacional de Educación 2010-2030 (componente ANEP): aportes para su elaboración (2010). Montevideo: opP; ANEP; NU; UNESCO, 2011. 
Uruguay: ¿Transformación o retorno? (Ensayo)

YARZÁBAL, L. Fundamentos teóricos de las políticas implementadas en el quinquenio. In: ANEP-CODICEN. Una transformación en marcha: políticas instrumentadas por el CODICEN 2005-2009. Montevideo: ANEP, 2010.

Recebido em março de 2011.

Aprovado em maio de 2011. 